Marcin Dudziński and KonRad Furmańczyk (Warszawa)

\title{
A NOTE ON CONTROL OF THE FALSE DISCOVERY PROPORTION
}

Abstract. We consider the problem of simultaneous testing of a finite number of null hypotheses $H_{i}, i=1, \ldots, s$. Starting from the classical paper of Lehmann (1957), it has become a very popular subject of research. In many applications, particularly in molecular biology (see e.g. Dudoit et al. (2003), Pollard et al. (2005)), the number $s$, i.e. the number of tested hypotheses, is large and the popular procedures that control the familywise error rate $(F W E R)$ have small power. Therefore, we are concerned with another error rate measure, called the false discovery proportion $(F D P)$. We prove some theorems about control of the FDP measure. Our results differ from those obtained by Lehmann and Romano (2005).

1. Introduction. In our paper, we consider the problem of simultaneous testing of a finite number of null hypotheses $H_{i}, i=1, \ldots, s$. Our main goal is to give some results concerning control of a measure, called the false discovery proportion (FDP). Suppose that data $X$ come from some probability distribution $P \in \Omega$, where $\Omega$ is the set of all available hypotheses (i.e., each single hypothesis $H_{i}$ is a certain subset $\omega_{i}$ of $\Omega$ ). Let $N$ denote the number of false rejections, and $R$ the total number of rejections. Then

$$
F D P:= \begin{cases}N / R & \text { if } R \neq 0, \\ 0 & \text { if } R=0 .\end{cases}
$$

Control of the FDP requires the following condition:

$$
P\{F D P>\gamma\} \leq \alpha \quad \text { for any } \gamma, \alpha \in(0 ; 1),
$$

for all possible constellations of true and false null hypotheses (i.e., for all $P \in \Omega)$.

2010 Mathematics Subject Classification: 62J15, 62G10.

Key words and phrases: false discovery proportion, multiple testing, Holm procedure, stepdown procedures, stepup procedures, $p$-value. 
2. Some procedures of control. In the first part of this section, we introduce the generalized Holm procedure, which we apply later in our proofs. In its second part, we present the Hochberg procedure. Before we introduce the generalized Holm procedure, we give the description of the Holm procedure in its general form (see also Holm (1979)). This procedure is described in terms of the $p$-values of individual tests.

Let us consider a single null hypothesis $H: P \in \omega$. To test $H_{i}: P \in \omega_{i}$, $i=1, \ldots, s$, we denote by $I(P)$ the set of indices of the true null hypotheses. Assume that $S_{\alpha}$, the rejection regions for a family of tests of $H$, indexed by $\alpha$, satisfy

$$
\begin{gathered}
P\left\{X \in S_{\alpha}\right\} \leq \alpha \quad \text { for all } 0<\alpha<1, P \in \omega, \\
S_{\alpha} \subset S_{\alpha^{\prime}} \quad \text { if } \alpha<\alpha^{\prime} .
\end{gathered}
$$

The $p$-value is defined by

$$
p=p(X):=\inf \left\{\alpha: X \in S_{\alpha}\right\} .
$$

Let $p_{1}, \ldots, p_{s}$ be the $p$-values of $s$ individual tests, let $p_{(1)} \leq \cdots \leq p_{(s)}$ denote these $p$-values ordered, and let $H_{(1)}, \ldots, H_{(s)}$ stand for the corresponding null hypotheses.

Put

$$
\alpha_{i}:=\alpha /(s-i+1) \quad \text { for some fixed } 0<\alpha<1, i=1, \ldots, s .
$$

The Holm procedure is described as follows: If

$$
p_{(1)}>\alpha_{1}
$$

we reject no null hypotheses. Otherwise, if

$$
p_{(1)} \leq \alpha_{1}, \ldots, p_{(r)} \leq \alpha_{r},
$$

we reject hypotheses $H_{(1)}, \ldots, H_{(r)}$, where the largest $r$ satisfying (5) is used.

The generalized Holm procedure has been introduced in Lehmann and Romano (2005). It is described similarly to the Holm procedure, with the $\alpha_{i}$ 's given by

$$
\alpha_{i}:=k \alpha /(s+k-i) \quad \text { for some } k \text { and some } 0<\alpha<1, i=1, \ldots, s .
$$

It turns out that the generalized Holm procedure with the $\alpha_{i}$ 's of the form

$$
\alpha_{i}:=\frac{([\gamma i]+1) \alpha}{s+[\gamma i]+1-i}, \quad i=1, \ldots, s,
$$

controls the FDP measure in the sense of (2) under the assumption $P\left\{q_{i} \leq u \mid r_{1}, \ldots, r_{s-|I(P)|}\right\} \leq u$ for any $i=1, \ldots,|I(P)|$ and any $u \in(0 ; 1)$, where $q_{1}, \ldots, q_{|I(P)|}$ denote the $p$-values corresponding to the $|I(P)|$ true null hypotheses, and $r_{1}, \ldots, r_{s-|I(P)|}$ are the $p$-values corresponding to the $s-|I(P)|$ false null hypotheses. For further details, see Theorem 3.1 in Lehmann and Romano (2005), together with its proof. 
Both the Holm procedure and the generalized Holm procedure are special cases of the so-called stepdown procedures. The stepdown procedures are described according to the steps (4) and (5), that is, a stepdown procedure starts with the most significant $p$-value and continues rejecting hypotheses as long as their corresponding $p$-values are small.

In the next part of this section, we present the Hochberg procedure (see Hochberg (1988)). It can be described as follows:

Let $\alpha_{i}$ be defined by (3). If

$$
p_{(s)} \leq \alpha_{s}
$$

we reject all null hypotheses. Otherwise, if

$$
p_{(s)}>\alpha_{s}, \ldots, p_{(r+1)}>\alpha_{r+1},
$$

we reject hypotheses $H_{(1)}, \ldots, H_{(r)}$, where $r$ denotes the smallest index satisfying (7).

The Hochberg procedure belongs to the class of so-called stepup procedures. A stepup procedure begins with the least significant $p$-value and continues accepting hypotheses as long as their corresponding $p$-values are large.

It is worth mentioning that it is the Hochberg procedure that is used in practice to control the FWER measure (recall that we define FWER as the probability of the event that at least one false rejection occurs). This procedure is more powerful, in the sense of average power, than the one proposed by Holm. However, the Holm procedure controls the FWER under no assumptions on the joint distribution of the $p$-values, whereas this is not so for the Hochberg procedure (see Romano and Shaikh (2006)).

3. Main results. Let, as previously, $|I(P)|=|I|$ denote the number of true hypotheses among $s$ null hypotheses $H_{i}, i=1, \ldots, s, q_{(1)} \leq \cdots \leq q_{(|I|)}$ be the ordered $p$-values corresponding to the $|I|$ true null hypotheses, and $r_{(1)} \leq \cdots \leq r_{(s-|I|)}$ denote the ordered $p$-values corresponding to the $s-|I|$ false null hypotheses.

Notice that, for the class of stepdown procedures (e.g., for the Holm procedures), the r.v.'s: $N$, the number of false rejections, and $T$, the number of true rejections, may be described (in terms of $p$-values) as follows:

$$
N=\left\{\begin{aligned}
& \mid\{k:\left.\exists p_{1 \leq i<s} p_{(1)} \leq \alpha_{1}, \ldots, p_{(i)} \leq \alpha_{i}, p_{(i+1)}>\alpha_{i+1} \wedge q_{(k)} \leq p_{(i)}<q_{(k+1)}\right\} \mid \\
& \text { if } q_{(|I|)}<p_{(s)}, \\
&|I| \quad \text { if } p_{(1)} \leq \alpha_{1}, \ldots, p_{(s)} \leq \alpha_{s} \text { and } q_{(|I|)}=p_{(s)}, \\
& 0 \quad \text { otherwise }
\end{aligned}\right.
$$




$$
T=\left\{\begin{array}{l}
\left|\left\{k: \underset{1 \leq i<s}{\exists} \quad p_{(1)} \leq \alpha_{1}, \ldots, p_{(i)} \leq \alpha_{i}, p_{(i+1)}>\alpha_{i+1} \wedge r_{(k)} \leq p_{(i)}<r_{(k+1)}\right\}\right| \\
\quad \text { if } r_{(s-|I|)}<p_{(s)}, \\
s-|I| \quad \text { if } p_{(1)} \leq \alpha_{1}, \ldots, p_{(s)} \leq \alpha_{s} \text { and } q_{(s-|I|)}=p_{(s)}, \\
0 \quad \text { otherwise, }
\end{array}\right.
$$

where $|\cdot|$ stands for cardinality.

We now formulate the main results of this paper. The proofs of Theorems 1-3, due to their length, are given in Appendix.

The first result concerns control of the FDP measure by using stepdown procedures.

THEOREM 1. Let $q_{1}, \ldots, q_{|I|}$ be identically distributed r.v.'s with marginal d.f. $F_{q}$ and $r_{1}, \ldots, r_{s-|I|}$ be identically distributed r.v.'s with marginal d.f. $F_{r}$. Suppose moreover that the sequence $\left\{q_{1}, \ldots, q_{|I|}\right\}$ is independent of the sequence $\left\{r_{1}, \ldots, r_{s-|I|}\right\}$, and that

$$
F_{q}(u) \leq u \quad \text { for all } u \in(0 ; 1) .
$$

Then any stepdown procedure with constants $\alpha_{1} \leq \cdots \leq \alpha_{s} \leq \alpha$ controls the FDP measure in (1) in the following sense:

(a) If $|I| \neq s$, then for any $0<\gamma<1$,

$$
\begin{aligned}
& P\{F D P>\gamma\} \\
& \leq \sum_{i l}\left\{\min \left(\frac{|I|}{l} \alpha_{i}, 1\right)-\max \left(\frac{|I| F_{q}\left(\alpha_{i}\right)-l}{|I|-l}, 0\right)\right\} \\
& \quad \times\left\{\min \left(\frac{s-|I|}{i-l} F_{r}\left(\alpha_{i}\right), 1\right)-\max \left(\frac{(s-|I|) F_{r}\left(\alpha_{i}\right)-(i-l)}{(s-|I|)-(i-l)}, 0\right)\right\} \\
& \quad+\sum_{i=1}^{|I|}\left(1-F_{r}\left(\alpha_{i+1}\right)\right) \min \left(\frac{|I|}{i} \alpha_{i}, 1\right) \\
& \quad+\sum_{i=|I|+1}^{\left[\frac{|I|-1}{\gamma}\right] \wedge(s-1)} \alpha_{i} \min \left(\frac{s-|I|}{s-i}\left(1-F_{r}\left(\alpha_{i+1}\right)\right), 1\right)+|I| \alpha_{s-|I|+1},
\end{aligned}
$$

where

$$
\sum_{i l}:=\sum_{i=1}^{\left[\frac{|I|-1}{\gamma}\right] \wedge s} \sum_{l=([\gamma i]+1) \vee(i-(s-|I|)+1)}^{(|I|-1) \wedge(i-1)} .
$$

(b) If $|I|=s$, then for any $0<\gamma<1$,

$$
P\{F D P>\gamma\} \leq \sum_{j=1}^{s} \frac{s}{j} \alpha_{j} .
$$


REMARK 1 (some numerical examples). Below, we present the values for the right hand side of (9) for the case when the generalized Holm procedure is applied with the $\alpha_{i}$ 's as in (6), where $F_{q}$ is the d.f. of the uniform distribution $U[0 ; 1]$, and $F_{r}$ is the d.f. of the beta distribution $B e(0.001,1)$.

For $s=100, \alpha=\alpha^{\prime} / 30$ with $\alpha^{\prime}=0.05, \gamma=0.05$ we have

\begin{tabular}{llllllllll}
\hline$|I|$ & 10 & 20 & 30 & 40 & 50 & 60 & 70 & 80 & 90 \\
r.h.s. & 0.02 & 0.04 & 0.05 & 0.05 & 0.05 & 0.04 & 0.03 & 0.02 & 0.01 \\
\hline
\end{tabular}

The results in the table above indicate control of the measure FDP at level $\alpha^{\prime}=0.05$, provided $\alpha$ in (6) is given by $\alpha=\alpha^{\prime} / 30=0.05 / 30$.

For $s=100, \alpha=\alpha^{\prime} / 40$ with $\alpha^{\prime}=0.05, \gamma=0.1$ we have

\begin{tabular}{llllllllll}
\hline$|I|$ & 10 & 20 & 30 & 40 & 50 & 60 & 70 & 80 & 90 \\
r.h.s. & 0.01 & 0.04 & 0.06 & 0.07 & 0.07 & 0.06 & 0.05 & 0.03 & 0.01 \\
\hline
\end{tabular}

For $s=1000, \alpha=\alpha^{\prime} / 400$ with $\alpha^{\prime}=0.05, \gamma=0.05$ we have

\begin{tabular}{llllllllll}
\hline$|I|$ & 100 & 200 & 300 & 400 & 500 & 600 & 700 & 800 & 900 \\
r.h.s. & 0.17 & 0.40 & 0.51 & 0.54 & 0.51 & 0.44 & 0.35 & 0.24 & 0.12 \\
\hline
\end{tabular}

For $s=1000, \alpha=\alpha^{\prime} / 400$ with $\alpha^{\prime}=0.05, \gamma=0.1$ we have

\begin{tabular}{llllllllll}
\hline$|I|$ & 100 & 200 & 300 & 400 & 500 & 600 & 700 & 800 & 900 \\
r.h.s. & 0.03 & 0.39 & 0.62 & 0.72 & 0.73 & 0.66 & 0.54 & 0.37 & 0.18 \\
\hline
\end{tabular}

The script which computes the right hand side of (9) is available at http:// mors.sggw.waw.pl/ kfurmanczyk/Theorem1.pdf.

In the case when the sequences $\left\{q_{n}\right\},\left\{r_{n}\right\}$ are i.i.d. and mutually independent, the following assertion can be proved.

TheOREM 2. Suppose that $\left\{q_{1}, \ldots, q_{|I|}\right\},\left\{r_{1}, \ldots, r_{s-|I|}\right\}$ are i.i.d. r.v.'s with marginal d.f's $F_{q}, F_{r}$, respectively, and all the assumptions of Theorem 1 hold. Then any stepdown procedure with constants $\alpha_{1} \leq \cdots \leq \alpha_{s} \leq \alpha$ controls the FDP measure in (1) in the following sense:

(a) If $|I| \neq s$, then for any $0<\gamma<1$,

$$
\begin{aligned}
& P\{F D P>\gamma\} \\
& \leq \sum_{i l}\left(\begin{array}{c}
|I| \\
l
\end{array}\right)\left(\alpha_{i}\right)^{l}\left(1-F_{q}\left(\alpha_{i}\right)\right)^{|I|-l}\left(\begin{array}{c}
s-|I| \\
i-l
\end{array}\right)\left(F_{r}\left(\alpha_{i}\right)\right)^{i-l}\left(1-F_{r}\left(\alpha_{i}\right)\right)^{s-|I|-i+l} \\
& \quad+\sum_{i=1}^{|I|}\left(1-F_{r}\left(\alpha_{i+1}\right)\right)^{s-|I|} \sum_{j=i}^{|I|}\left(\begin{array}{c}
|I| \\
j
\end{array}\right)\left(\alpha_{i}\right)^{j}\left(1-F_{q}\left(\alpha_{i}\right)\right)^{|I|-j}
\end{aligned}
$$




$$
\begin{aligned}
& +\sum_{i=|I|+1}^{\left[\frac{|I|-1}{\gamma}\right] \wedge(s-1)}\left(\alpha_{i}\right)^{|I|} \sum_{j=0}^{i-|I|}\left(\begin{array}{c}
s-|I| \\
j
\end{array}\right)\left(F_{r}\left(\alpha_{i+1}\right)\right)^{j}\left(1-F_{r}\left(\alpha_{i+1}\right)\right)^{s-|I|-j} \\
& +\sum_{i=s-|I|+1}^{\left[\frac{|I|-1}{\gamma}\right] \wedge(s-1)}\left(F_{r}\left(\alpha_{i}\right)\right)^{s-|I|}\left(\begin{array}{c}
|I| \\
i-s+|I|
\end{array}\right)\left(\alpha_{i}\right)^{i-s+|I|}\left(1-F_{q}\left(\alpha_{i}\right)\right)^{s-i} \\
& +\left(F_{r}\left(\alpha_{s}\right)\right)^{s-|I|}\left(\alpha_{s}\right)^{|I|},
\end{aligned}
$$

where $\sum_{i l}$ is as in Theorem 1.

(b) If $|I|=s$, then for any $0<\gamma<1$,

$$
P\{F D P>\gamma\} \leq \sum_{i=1}^{s} \sum_{j=i}^{s}\left(\begin{array}{l}
s \\
j
\end{array}\right)\left(\alpha_{i}\right)^{j}\left(1-F_{q}\left(\alpha_{i}\right)\right)^{s-j} .
$$

REMARK 2 (some numerical examples). Below, we give the values for the right hand side of (11) for the case when the generalized Holm procedure is applied with the $\alpha_{i}$ 's as in (6), where $\alpha=0.05, F_{q}$ is the d.f. of the uniform distribution $U[0 ; 1]$, and $F_{r}$ is the d.f. of the beta distribution $\operatorname{Be}(0.01,1)$.

For $s=100, \gamma=0.05$ we have

\begin{tabular}{llllllllll}
\hline$|I|$ & 10 & 20 & 30 & 40 & 50 & 60 & 70 & 80 & 90 \\
r.h.s. & 0.004 & 0.005 & 0.005 & 0.007 & 0.008 & 0.014 & 0.018 & 0.048 & 0.072 \\
\hline
\end{tabular}

For $s=100, \gamma=0.1$ we have

\begin{tabular}{llllllllll}
\hline$|I|$ & 10 & 20 & 30 & 40 & 50 & 60 & 70 & 80 & 90 \\
r.h.s. & $6 \mathrm{e}-15$ & 0.010 & 0.012 & 0.014 & 0.018 & 0.023 & 0.030 & 0.040 & 0.060 \\
\hline
\end{tabular}

For $s=1000, \gamma=0.05$ we have

\begin{tabular}{llllllllll}
\hline$|I|$ & 100 & 200 & 300 & 400 & 500 & 600 & 700 & 800 & 900 \\
r.h.s. & $5 \mathrm{e}-17$ & $2 \mathrm{e}-17$ & $\mathrm{e}-16$ & $2 \mathrm{e}-15$ & $7 \mathrm{e}-15$ & $5 \mathrm{e}-12$ & $8 \mathrm{e}-10$ & $2 \mathrm{e}-7$ & $9 \mathrm{e}-15$
\end{tabular}

For $s=1000, \gamma=0.1$ we have

\begin{tabular}{llllllllll}
\hline$|I|$ & 100 & 200 & 300 & 400 & 500 & 600 & 700 & 800 & 900 \\
r.h.s. & $3 \mathrm{e}-15$ & $2 \mathrm{e}-15$ & $7 \mathrm{e}-15$ & $8 \mathrm{e}-14$ & $2 \mathrm{e}-12$ & $9 \mathrm{e}-11$ & $7 \mathrm{e}-9$ & $1 \mathrm{e}-6$ & $3 \mathrm{e}-4$ \\
\hline
\end{tabular}

We have obtained similar results for the case when $F_{q} \sim U[0 ; 1], F_{r} \sim$ $B e(0.05,1)$.

It is worth mentioning that a good estimation of the FDP measure can lead to a better control of this measure.

The script which computes the right hand side of (11) is available at http://mors.sggw.waw.pl/ kfurmanczyk/Theorem2.pdf.

Our next result is the following. 

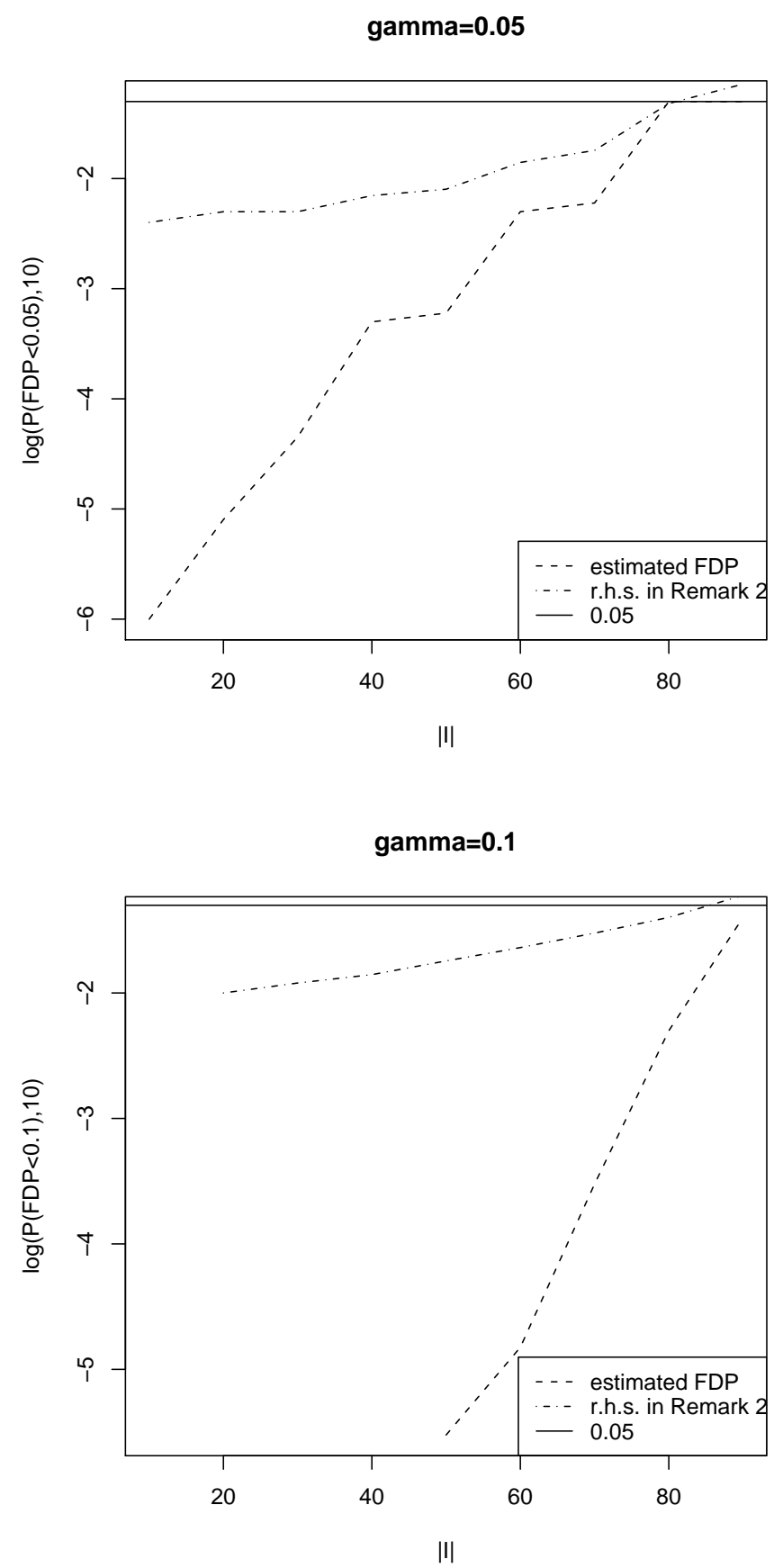

Fig. 1. Comparison of the r.h.s. in (11) with the simulated values of FDP; the cases when $\gamma=0.05$ and $\gamma=0.01$. 
TheOREM 3. Suppose that $q_{1}, \ldots, q_{|I|}$ are identically distributed r.v.'s with marginal d.f. $F_{q}$ and (8) holds. Then any stepdown procedure with constants $\alpha_{1} \leq \cdots \leq \alpha_{s} \leq \alpha$ controls the FDP measure in (1) in the following sense:

For any $0<\gamma<1$,

$$
P\{F D P>\gamma\} \leq \sum_{j=1}^{|I|} \frac{|I|}{j} \alpha_{j}+\frac{|I|}{C(\gamma)} \alpha_{s-|I|+C(\gamma)},
$$

where $C(\gamma):=\min ([\gamma /(1-\gamma)]+1,|I|)$.

REMARK 3 (some numerical examples). Below, we present the values of the right hand side of (13) for the case when the generalized Holm procedure is applied with the $\alpha_{i}$ 's as in (6), where $F_{q}$ is the d.f. of the uniform distribution $U[0 ; 1]$.

For $s=100, \alpha=\alpha^{\prime} / 20$ with $\alpha^{\prime}=0.05, \gamma=0.05$ we have

\begin{tabular}{llllllllll}
\hline$|I|$ & 10 & 20 & 30 & 40 & 50 & 60 & 70 & 80 & 90 \\
r.h.s. & 0.01 & 0.01 & 0.01 & 0.02 & 0.02 & 0.02 & 0.02 & 0.03 & 0.04 \\
\hline
\end{tabular}

For $s=100, \alpha=\alpha^{\prime} / 25$ with $\alpha^{\prime}=0.05, \gamma=0.1$ we have

\begin{tabular}{llllllllll}
\hline$|I|$ & 10 & 20 & 30 & 40 & 50 & 60 & 70 & 80 & 90 \\
r.h.s. & 0.01 & 0.01 & 0.02 & 0.02 & 0.02 & 0.02 & 0.03 & 0.03 & 0.04 \\
\hline
\end{tabular}

For $s=1000, \alpha=\alpha^{\prime} / 100$ with $\alpha^{\prime}=0.05, \gamma=0.05$ we have

\begin{tabular}{llllllllll}
\hline$|I|$ & 100 & 200 & 300 & 400 & 500 & 600 & 700 & 800 & 900 \\
r.h.s. & 0.02 & 0.02 & 0.02 & 0.02 & 0.02 & 0.02 & 0.03 & 0.04 & 0.05 \\
\hline
\end{tabular}

For $s=1000, \alpha=\alpha^{\prime} / 180$ with $\alpha^{\prime}=0.05, \gamma=0.1$ we have

\begin{tabular}{llllllllll}
\hline$|I|$ & 100 & 200 & 300 & 400 & 500 & 600 & 700 & 800 & 900 \\
r.h.s. & 0.01 & 0.02 & 0.02 & 0.02 & 0.02 & 0.03 & 0.03 & 0.04 & 0.05 \\
\hline
\end{tabular}

The script which computes the right hand side of (13) is available at http:// mors.sggw.waw.pl/ kfurmanczyk/Theorem3.pdf.

The result below follows immediately from Theorem 3 .

Corollary 1. Let

$$
\alpha_{i}:=\frac{C(\gamma) \alpha}{s+C(\gamma)-i}, \quad i=1, \ldots, s, \quad C(\gamma):=\min \left(\left[\frac{\gamma}{1-\gamma}\right]+1,|I|\right) .
$$

Then, for the generalized Holm procedure with the $\alpha_{i}$ 's given by (14), the conclusion in (13) holds.

We now introduce a new stepdown procedure which controls the FDP. Using this procedure requires the $\mathrm{MTP}_{2}$ (multivariate totally positive of 
order two) dependence assumption for the $p$-values for true null hypotheses. An $n$-dimensional random vector is said to have an $\mathrm{MTP}_{2}$ distribution if the corresponding probability density function $f(x)$ satisfies

$$
f(x \vee y) f(x \wedge y) \geq f(x) f(y) \quad \text { for all } x, y \in \mathbb{R}^{n},
$$

where $x=\left(x_{1}, \ldots, x_{n}\right), y=\left(y_{1}, \ldots, y_{n}\right)$,

$$
\begin{aligned}
& x \vee y=\left(\max \left(x_{1}, y_{1}\right), \ldots, \max \left(x_{n}, y_{n}\right)\right), \\
& x \wedge y=\left(\min \left(x_{1}, y_{1}\right), \ldots, \min \left(x_{n}, y_{n}\right)\right) .
\end{aligned}
$$

We prove the following result.

Proposition 1. Suppose that $\left(q_{1}, \ldots, q_{|I|}\right)$ is an $M T P_{2}$ random vector and let $\alpha_{1} \leq \cdots \leq \alpha_{s} \leq \alpha$ denote a numerical sequence such that $\left(\alpha_{i} / i\right)_{i=1}^{M}$, where $M:=\min ([\gamma s]+1,|I|)$, is nondecreasing. Then the stepdown procedure with critical values $\alpha_{i}$ controls the FDP in (1) at level $\alpha$.

Proof. Observe that, for any given $\gamma \in(0 ; 1)$,

$$
\begin{aligned}
& P\{F D P>\gamma\} \\
& \quad=P\{F D P>\gamma, R>0\}=P\left\{\bigcup_{i=1}^{s}\{N / R>\gamma, R=i\}\right\} \\
& \quad=P\left\{\bigcup_{i=1}^{s}\{N>\gamma i, R=i\}\right\}=P\left\{\bigcup_{i=1}^{s}\{N \geq[\gamma i]+1, R=i\}\right\} .
\end{aligned}
$$

For fixed $i$, let $j(i)$ denote the smallest index such that $q_{([\gamma i]+1)}=p_{(j(i))}$. Since the event $\{N \geq[\gamma i]+1\}$ is a subset of $\left\{q_{([\gamma i]+1)} \leq \alpha_{j(i)}\right\}$, we have

$$
P\left\{\bigcup_{i=1}^{s}\{N \geq[\gamma i]+1, R=i\}\right\} \leq P\left\{\bigcup_{i=1}^{s}\left\{q_{([\gamma i]+1)} \leq \alpha_{j(i)}, R=i\right\}\right\} .
$$

It follows from the definition of $j(i)$ that $j(i) \leq[\gamma i]+1+s-|I|$ (see (13) in Lehmann and Romano (2005)). Thus, we obtain

$$
\alpha_{j(i)} \leq \alpha_{[\gamma i]+1+s-|I|} \cdot
$$

For fixed $i$, let $w:=[\gamma i]+1$. It follows from (15)-(17) that

$$
\begin{aligned}
P\{F D P>\gamma\} & \leq P\left\{\bigcup_{i=1}^{s}\left\{q_{([\gamma i]+1)} \leq \alpha_{[\gamma i]+1+s-|I|}\right\}\right\} \\
& \leq P\left\{\bigcup_{w=1}^{M}\left\{q_{(w)} \leq \alpha_{w+s-|I|}\right\}\right\},
\end{aligned}
$$

where $M:=\min ([\gamma s]+1,|I|)$. Since, in addition, $\left(q_{1}, \ldots, q_{M}\right)$ is an $M T P_{2}$ random vector and the sequence $\left(\alpha_{i} / i\right)_{i=1}^{M}$ is nondecreasing, by Theorem 
3.1(i) in Sarkar (1998) we have

$$
P\left\{q_{(1)} \geq \alpha_{1+s-|I|}, \ldots, q_{(M)} \geq \alpha_{M+s-|I|}\right\} \geq 1-\frac{1}{M} \sum_{w=1}^{M} \alpha_{w+s-|I|} .
$$

Hence,

$$
\begin{aligned}
& P\left\{\bigcup_{w=1}^{M}\left\{q_{(w)}<\alpha_{w+s-|I|}\right\}\right\} \\
= & 1-P\left\{q_{(1)} \geq \alpha_{1+s-|I|}, \ldots, q_{(M)} \geq \alpha_{M+s-|I|}\right\} \leq \frac{1}{M} \sum_{w=1}^{M} \alpha_{w+s-|I|} \leq \alpha .
\end{aligned}
$$

Due to (18), (19), we obtain

$$
P\{F D P>\gamma\} \leq \alpha,
$$

as asserted.

EXAMPLE. Suppose that we are testing the null hypothesis $H_{i}: \mu_{i}=0$ against the alternative $H_{i}^{\prime}: \mu_{i}>0$, where $\mu_{i}$ is the mean of the normal distribution. Let $p_{i}=1-\Phi\left(T_{i}\right)$, where $\Phi$ stands for the standard normal d.f. and the $T_{i}$ 's denote the standard normal, positively correlated test statistics. Then $\left(p_{1}, \ldots, p_{s}\right)$ is an $\mathrm{MTP}_{2}$ random vector (for details see Sarkar (1998)), and Proposition 1 may be applied.

We now prove the following statement.

Proposition 2. Suppose that (8) is satisfied. Then, for any stepdown procedure with constants $\alpha_{1} \leq \cdots \leq \alpha_{s} \leq \alpha$, we have

$$
P\{F D P>\gamma\} \leq|I| \beta_{1}+|I| \sum_{j=2}^{M} \frac{\beta_{j}-\beta_{j-1}}{j} \quad \text { for any } 0<\gamma<1,
$$

where

$$
\begin{aligned}
\beta_{j} & := \begin{cases}\min \left(\alpha_{j+s-|I|}, \alpha_{[(j-1) / \gamma]+1}\right) & \text { if }[(j-1) / \gamma]+1 \leq s, \\
\alpha_{j+s-|I|} & \text { otherwise, }\end{cases} \\
M & :=\min ([\gamma s]+1,|I|) .
\end{aligned}
$$

Proof. Recall that, by (15), (16),

$$
P\{F D P>\gamma\} \leq P\left\{\bigcup_{i=1}^{s}\left\{q_{([\gamma i]+1)} \leq \alpha_{j(i)}, R=i\right\}\right\},
$$

where $j(i)$ denotes the smallest index such that $q_{([\gamma i]+1)}=p_{(j(i))}$. Therefore (see also (17)), we can write 


$$
\begin{aligned}
P\{F D P>\gamma\} & \leq P\left\{\bigcup_{i=1}^{s}\left\{q_{([\gamma i]+1)} \leq \min \left(\alpha_{[\gamma i]+1+s-|I|}, \alpha_{i}\right)\right\}\right\} \\
& \leq P\left\{\bigcup_{j=1}^{M}\left\{q_{(j)} \leq \min \left(\alpha_{j+s-|I|}, \alpha_{[(j-1) / \gamma]+1}\right)\right\}\right\} \\
& =P\left\{\bigcup_{j=1}^{M}\left\{q_{(j)} \leq \beta_{j}\right\}\right\},
\end{aligned}
$$

where $\beta_{j}, M$ are defined by (21), (22), respectively. Observe that the sequence $\left\{\beta_{j}\right\}$ is nondecreasing. This fact together with relation (23) and Lemma 3.1 in Lehmann and Romano (2005) yield (20).

Corollary 2. Suppose that $M$ and the $\beta_{j}$ 's are as in Proposition 2 and (8) holds. Put

$$
\begin{aligned}
S_{1}(s,|I|, \gamma) & :=|I| \beta_{1}+|I| \sum_{j=2}^{M} \frac{\beta_{j}-\beta_{j-1}}{j}, \\
D_{1}(s, \gamma) & :=\max _{1 \leq|I| \leq s} S_{1}(s,|I|, \gamma), \quad \beta_{j}^{\prime}:=\alpha \beta_{j} / D_{1}(s, \gamma) .
\end{aligned}
$$

Then, for any stepdown procedure with critical values of the form $\alpha_{i}^{\prime}=$ $\alpha \alpha_{i} / D_{1}(s, \gamma)$, we have for any $0<\gamma<1$,

$$
P\{F D P>\gamma\} \leq \alpha .
$$

Proof. By Proposition 2, with the critical values $\alpha_{i}^{\prime}$, we obtain

$$
\begin{aligned}
P\{F D P & >\gamma\} \leq|I| \beta_{1}^{\prime}+|I| \sum_{j=2}^{M} \frac{\beta_{j}^{\prime}-\beta_{j-1}^{\prime}}{j} \\
& =\frac{\alpha}{D_{1}(s, \gamma)}\left(|I| \beta_{1}+|I| \sum_{j=2}^{M} \frac{\beta_{j}-\beta_{j-1}}{j}\right)=\frac{\alpha S_{1}(s,|I|, \gamma)}{D_{1}(s, \gamma)} \leq \alpha,
\end{aligned}
$$

as desired.

REMARK 4 (some numerical examples). The tables below give the values of $S_{1}(s,|I|, \gamma)$ for the case when the generalized Holm procedure is applied with the $\alpha_{i}^{\prime} s$ as in (6) and $\alpha=0.05$.

For $s=100, \gamma=0.05$ we have

\begin{tabular}{llllllllll}
\hline$|I|$ & 10 & 20 & 30 & 40 & 50 & 60 & 70 & 80 & 90 \\
$S_{1}(\cdot)$ & 0.69 & 1.23 & 1.39 & 1.55 & 1.45 & 1.57 & 1.33 & 1.42 & 0.97 \\
\hline
\end{tabular}

For $s=100, \gamma=0.1$ we have

\begin{tabular}{llllllllll}
\hline$|I|$ & 10 & 20 & 30 & 40 & 50 & 60 & 70 & 80 & 90 \\
$S_{1}(\cdot)$ & 0.82 & 1.63 & 1.81 & 1.94 & 2.01 & 2.02 & 1.97 & 1.84 & 1.59 \\
\hline
\end{tabular}


For $s=1000, \gamma=0.05$ we have

\begin{tabular}{llllllllll}
\hline$|I|$ & 100 & 200 & 300 & 400 & 500 & 600 & 700 & 800 & 900 \\
$S_{1}(\cdot)$ & 1.57 & 1.98 & 2.31 & 2.58 & 2.78 & 2.90 & 2.93 & 2.81 & 2.43 \\
\hline
\end{tabular}

For $s=1000, \gamma=0.1$ we have

\begin{tabular}{llllllllll}
\hline$|I|$ & 100 & 200 & 300 & 400 & 500 & 600 & 700 & 800 & 900 \\
$S_{1}(\cdot)$ & 1.54 & 2.18 & 2.60 & 2.96 & 3.24 & 3.45 & 3.55 & 3.52 & 3.19 \\
\hline
\end{tabular}

The results above indicate that $D_{1}(100,0.05)=1.57, D_{1}(100,0.1)=2.02$, $D_{1}(1000,0.05)=2.93, D_{1}(1000,0.1)=3.55$. The relevant script is available at http://mors.sggw.waw.pl/ kfurmanczyk/Remark4.pdf.
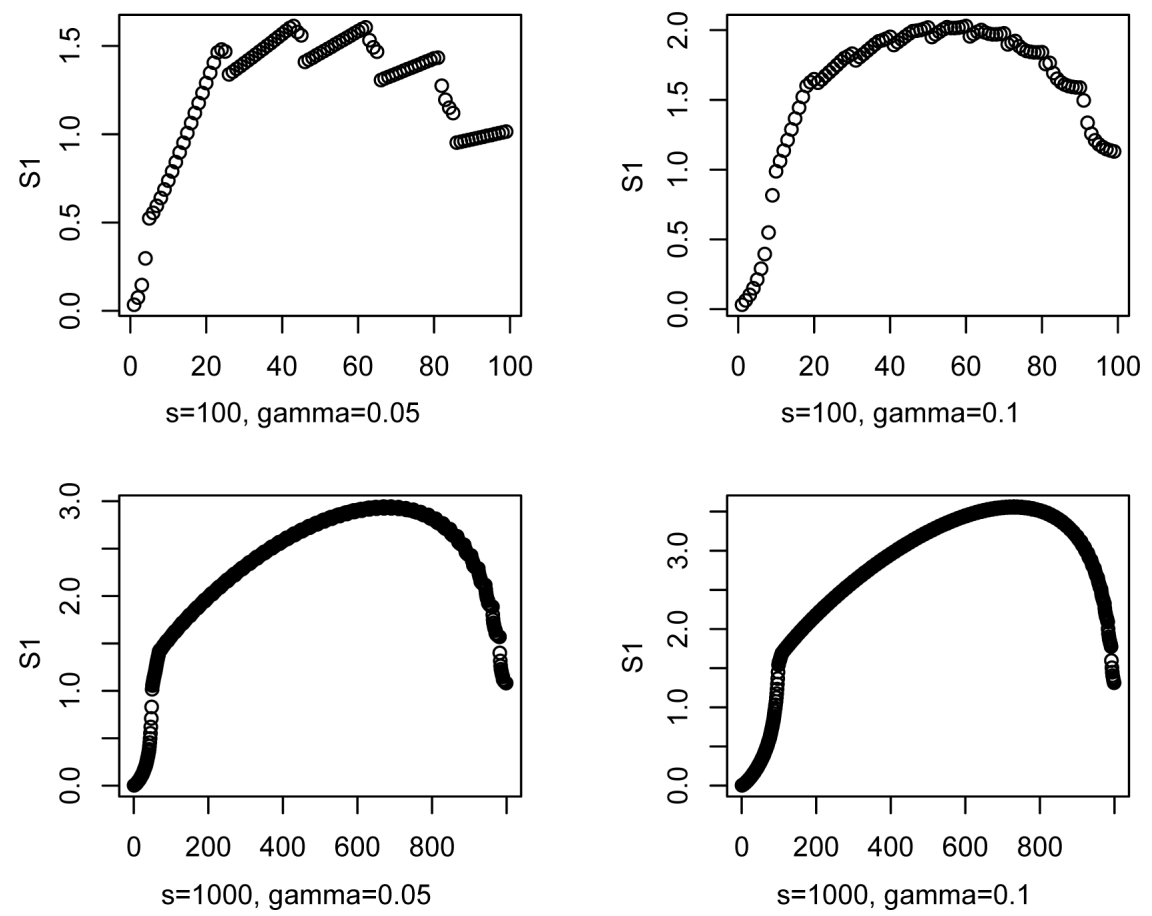

Fig. 2. The values of $S_{1}$ for the generalized Holm procedure; the cases when $s=100$ and $\gamma=0.05, s=100$ and $\gamma=0.1, s=1000$ and $\gamma=0.05, s=1000$ and $\gamma=0.1$.

REMARK 5. By Theorem 3.3 of Lehmann and Romano (2005), we obtain control of the FDP at level $\alpha$ by using the generalized Holm procedure with the critical values $\alpha_{i}^{\prime} \sim \alpha_{i} / \log ([\gamma s]+1)$, where the $\alpha_{i}^{\prime}$ 's are as in (6). Observe that: 1$)$ for $s=100$ and $\gamma=0.05$, we get $\log ([\gamma s]+1) \approx 1.79,2)$ for $s=100$ and $\gamma=0.1$, we obtain $\log ([\gamma s]+1) \approx 2.40,3)$ for $s=1000$ and $\gamma=0.05$, we get $\log ([\gamma s]+1) \approx 3.93,4)$ for $s=1000$ and $\gamma=0.1$, we obtain $\log ([\gamma s]+1) \approx 4.62$. These calculations show that the norming constants 
$D_{1}(s, \gamma)$ we have used in Corollary 2 are smaller than the norming constants $C_{[\gamma s]+1} \sim \log ([\gamma s]+1)$, applied in Lehmann and Romano (2005).

4. Appendix. In this section, we give the proofs of our main results, as well as some auxiliary results.

Proof of Theorem 1(a). Assume that $|I|>0$, as otherwise there is nothing to prove.

Notice that

$$
\begin{aligned}
& P\{F D P>\gamma\}=\sum_{i=1}^{s} P\{N / R>\gamma, R=i\} \\
& \quad=\sum_{i=1}^{s} P\left\{\frac{N}{N+T}>\gamma, N+T=i\right\}=\sum_{i=1}^{s} P\{N>\gamma i, N+T=i\} .
\end{aligned}
$$

Hence,

$$
\begin{aligned}
P\{F D P>\gamma\} & =\sum_{i=1}^{\left[\frac{|I|-1}{\gamma}\right] \wedge s} P\{N \geq[\gamma i]+1, N+T=i\} \\
& =\sum_{i=1}^{\left[\frac{|I|-1}{\gamma}\right] \wedge s} \sum_{l=[\gamma i]+1}^{|I| \wedge i} P\{N \geq[\gamma i]+1, N+T=i, N=l\},
\end{aligned}
$$

and we can write

$$
\begin{aligned}
& P\{F D P>\gamma\} \\
& \leq \sum_{i=1}^{\left[\frac{|I|-1}{\gamma}\right] \wedge s} \sum_{l=[\gamma i]+1}^{|I| \wedge i} P\{N+T=i, N=l\}=\sum_{i=1}^{\left[\frac{|I|-1}{\gamma}\right] \wedge s} \sum_{l=[\gamma i]+1}^{|I| \wedge i} P\{T=i-l, N=l\}
\end{aligned}
$$

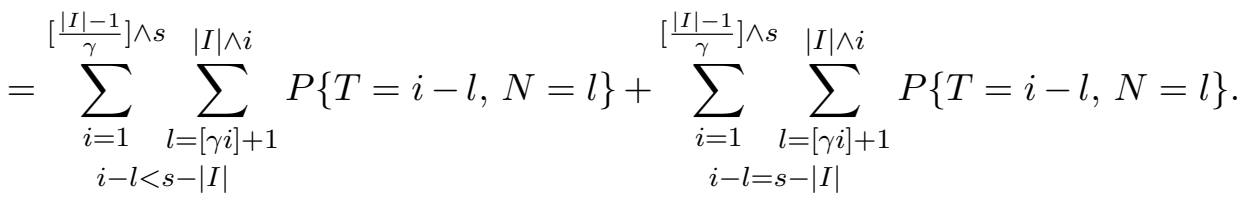

Let us consider the following cases:

$$
\begin{aligned}
& 1^{\circ} 0<i-l<s-|I| \text { and } 0<l<|I|, \\
& 2^{\mathrm{o}} i-l=0, \\
& 3^{\mathrm{o}} 0<i-l<s-|I| \text { and } l=|I|, \\
& 4^{\mathrm{o}} i-l=s-|I| .
\end{aligned}
$$

Notice that $i-l<s-|I|$ implies $l \geq i-(s-|I|)+1$. Thus, 


$$
\begin{aligned}
P\{F D P>\gamma\} \leq & \sum_{i=1}^{\left[\frac{|I|-1}{\gamma}\right] \wedge s} \sum_{l=([\gamma i]+1) \vee(i-(s-|I|)+1)}^{(|I|-1) \wedge(i-1)} P\{T=i-l, N=l\} \\
& +\sum_{i=1}^{|I|} P\{T=0, N=i\} \\
& +\sum_{i=|I|+1}^{\left[\frac{|I|-1}{\gamma}\right] \wedge s} P\{T=i-|I|, N=|I|\} \\
& +\sum_{i=1}^{\left[\frac{|I|-1}{\gamma}\right] \wedge s} \sum_{\substack{l I \mid \wedge i \\
l=l}} P\{T=s-|I|+1
\end{aligned}
$$

Observe that, provided (see case $1^{\circ}$ ) $0<i-l<s-|I|$ and $0<l<|I|$, we obtain

$$
\begin{aligned}
P\{N= & l, T=i-l\} \\
& \leq P\left\{q_{(l)} \leq \alpha_{i}, q_{(l+1)}>\alpha_{i}, r_{(i-l)} \leq \alpha_{i}, r_{(i-l+1)}>\alpha_{i}\right\} \\
& =P\left\{q_{(l)} \leq \alpha_{i}, q_{(l+1)}>\alpha_{i}\right\} P\left\{r_{(i-l)} \leq \alpha_{i}, r_{(i-l+1)}>\alpha_{i}\right\},
\end{aligned}
$$

where the last relation follows from the assumption that $\left\{q_{n}\right\}$ is independent of $\left\{r_{n}\right\}$. Notice that

$$
\begin{aligned}
P\left\{q_{(l)} \leq \alpha_{i}, q_{(l+1)}>\alpha_{i}\right\} & =P\left\{q_{(l)} \leq \alpha_{i}\right\}-P\left\{q_{(l)} \leq \alpha_{i}, q_{(l+1)} \leq \alpha_{i}\right\} \\
& =P\left\{q_{(l)} \leq \alpha_{i}\right\}-P\left\{q_{(l+1)} \leq \alpha_{i}\right\}
\end{aligned}
$$

By Proposition A below and assumption (8), we have

$$
P\left\{q_{(l)} \leq \alpha_{i}\right\} \leq \min \left(\frac{|I|}{l} F_{q}\left(\alpha_{i}\right), 1\right) \leq \min \left(\frac{|I|}{l} \alpha_{i}, 1\right) .
$$

Furthermore, due to Proposition B below, we obtain

$$
P\left\{q_{(l+1)} \leq \alpha_{i}\right\} \geq \max \left(1-\frac{|I|}{|I|-l}\left(1-F_{q}\left(\alpha_{i}\right)\right), 0\right) .
$$

Thus, by (27)-(29),

$$
\begin{aligned}
P\left\{q_{(l)} \leq \alpha_{i}, q_{(l+1)}>\alpha_{i}\right\} & \\
& \leq \min \left(\frac{|I|}{l} \alpha_{i}, 1\right)-\max \left(1-\frac{|I|}{|I|-l}\left(1-F_{q}\left(\alpha_{i}\right)\right), 0\right) .
\end{aligned}
$$

In addition, by identical reasoning to that in (27), we get

$$
P\left\{r_{(i-l)} \leq \alpha_{i}, r_{(i-l+1)}>\alpha_{i}\right\}=P\left\{r_{(i-l)} \leq \alpha_{i}\right\}-P\left\{r_{(i-l+1)} \leq \alpha_{i}\right\} .
$$


It follows from Proposition A that

$$
P\left\{r_{(i-l)} \leq \alpha_{i}\right\} \leq \min \left(\frac{s-|I|}{i-l} F_{r}\left(\alpha_{i}\right), 1\right) .
$$

Moreover, due to Proposition B,

$$
P\left\{r_{(i-l+1)} \leq \alpha_{i}\right\} \geq \max \left(1-\frac{s-|I|}{s-|I|-i+l}\left(1-F_{r}\left(\alpha_{i}\right)\right), 0\right) .
$$

The relations (31)-(33) imply

$$
\begin{aligned}
& P\left\{r_{(i-l)} \leq \alpha_{i}, r_{(i-l+1)}>\alpha_{i}\right\} \\
\leq & \min \left(\frac{s-|I|}{i-l} F_{r}\left(\alpha_{i}\right), 1\right)-\max \left(1-\frac{s-|I|}{s-|I|-i+l}\left(1-F_{r}\left(\alpha_{i}\right)\right), 0\right) .
\end{aligned}
$$

By (26), (30) and (34), we obtain

(35) $\quad P\{T=i-l, N=l\}$

$$
\begin{aligned}
\leq & \left\{\min \left(\frac{|I|}{l} \alpha_{i}, 1\right)-\max \left(1-\frac{|I|}{|I|-l}\left(1-F_{q}\left(\alpha_{i}\right)\right), 0\right)\right\} \\
& \times\left\{\min \left(\frac{s-|I|}{i-l} F_{r}\left(\alpha_{i}\right), 1\right)-\max \left(1-\frac{s-|I|}{s-|I|-i+l}\left(1-F_{r}\left(\alpha_{i}\right)\right), 0\right)\right\} \\
= & \left\{\min \left(\frac{|I|}{l} \alpha_{i}, 1\right)-\max \left(\frac{|I| F_{q}\left(\alpha_{i}\right)-l}{|I|-l}, 0\right)\right\} \\
& \times\left\{\min \left(\frac{s-|I|}{i-l} F_{r}\left(\alpha_{i}\right), 1\right)-\max \left(\frac{(s-|I|) F_{r}\left(\alpha_{i}\right)-(i-l)}{(s-|I|)-(i-l)}, 0\right)\right\}
\end{aligned}
$$

if $0<i-l<s-|I|$ and $0<l<|I|$.

We now consider case $2^{\circ}$. Then $i=l$ and $0<i \leq|I|<s$ (as $l \leq|I|$ and $|I| \neq s)$. Under these conditions, we get

$$
\begin{aligned}
P\{T=i-l, N=l\} & =P\{N=i, T=0\} \\
& \leq P\left\{q_{(i)} \leq \alpha_{i}, r_{(1)}>\alpha_{i+1}\right\} \\
& =P\left\{q_{(i)} \leq \alpha_{i}\right\} P\left\{r_{(1)}>\alpha_{i+1}\right\} \\
& \leq P\left\{q_{(i)} \leq \alpha_{i}\right\} P\left\{r_{1}>\alpha_{i+1}\right\} \\
& =P\left\{q_{(i)} \leq \alpha_{i}\right\}\left(1-P\left\{r_{1} \leq \alpha_{i+1}\right\}\right) .
\end{aligned}
$$

The derivation in (36), Proposition A and assumption (8) imply

$$
P\{T=0, N=i\} \leq\left(1-F_{r}\left(\alpha_{i+1}\right)\right) \min \left(\frac{|I|}{i} \alpha_{i}, 1\right) .
$$

Next, we assume that $0<i-l<s-|I|$ and $l=|I|$ (see $3^{\circ}$ ). Then $|I|<i<s, 0<i-|I|<s-|I|$, and 
(38)

$$
\begin{aligned}
P\{T=i-l, N=l\} & =P\{N=|I|, T=i-|I|\} \\
& \leq P\left\{q_{(|I|)} \leq \alpha_{i}, r_{(i-|I|+1)}>\alpha_{i+1}\right\} \\
& =P\left\{q_{(|I|)} \leq \alpha_{i}\right\} P\left\{r_{(i-|I|+1)}>\alpha_{i+1}\right\} \\
& =P\left\{q_{(|I|)} \leq \alpha_{i}\right\}\left(1-P\left\{r_{(i-|I|+1)} \leq \alpha_{i+1}\right\}\right) .
\end{aligned}
$$

The derivation in (38), Propositions A, B and assumption (8) yield

$$
\begin{aligned}
P\{T=i-|I|, N & =|I|\} \\
& \leq \alpha_{i}\left(1-\max \left(1-\frac{s-|I|}{s-i}\left(1-F_{r}\left(\alpha_{i+1}\right)\right), 0\right)\right) \\
& =\alpha_{i} \min \left(\frac{s-|I|}{s-i}\left(1-F_{r}\left(\alpha_{i+1}\right)\right), 1\right) .
\end{aligned}
$$

We now suppose that condition $4^{\mathrm{o}}$ is satisfied, i.e. $i-l=s-|I|$. Then

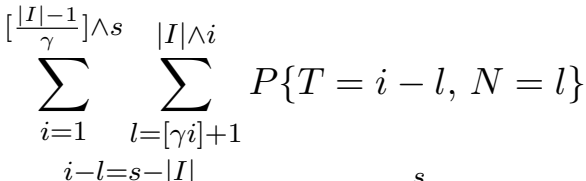

$$
\begin{aligned}
& \leq \sum_{i=(s-|I|)+1}^{s} P\{T=s-|I|, N=i-(s-|I|)\} \\
& \leq \sum_{l=1}^{|I|} P\{N=l\}=P\{N \geq 1\} \leq P\left\{q_{(1)} \leq \alpha_{j}\right\},
\end{aligned}
$$

where $j$ is the smallest index satisfying $p_{(j)}=q_{(1)}$. Since (see (13) in Lehmann and Romano (2005))

$$
1 \leq j \leq s-|I|+1,
$$

we obtain $P\left\{q_{(1)} \leq \alpha_{j}\right\} \leq P\left\{q_{(1)} \leq \alpha_{s-|I|+1}\right\}$, and consequently, by (40),

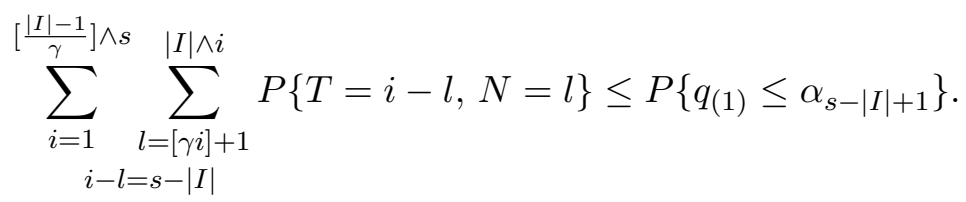

It follows from Proposition A and assumption (8) that

$$
P\left\{q_{(1)} \leq \alpha_{s-|I|+1}\right\} \leq|I| F_{q}\left(\alpha_{s-|I|+1}\right) \leq|I| \alpha_{s-|I|+1} .
$$

Thus, due to (41), (42),

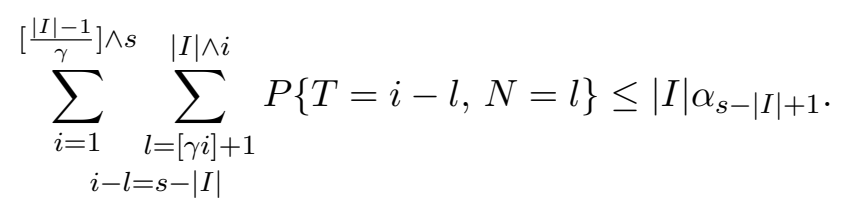

The relations (25), (35), (37), (39) and (43) yield (9). 
Proof of Theorem 1(b). Observe that, provided $|I|=s$, we have

$$
\begin{aligned}
P\{F D P>\gamma\} & =P\{F D P>\gamma, T=0\} \\
& =P\{1>\gamma, T=0, R>0\}=P\{T=0, R>0\} \\
& =\sum_{j=1}^{s} P\{T=0, R=j\} .
\end{aligned}
$$

Furthermore, notice that, for $j \in\{1, \ldots, s\}$,

$$
P\{T=0, R=j\}=P\{N=j, R=j\} \leq P\left\{q_{(j)} \leq \alpha_{j}\right\} .
$$

By Proposition A and assumption (8), we conclude that

$$
P\left\{q_{(j)} \leq \alpha_{j}\right\} \leq \frac{s}{j} F_{q}\left(\alpha_{j}\right) \leq \frac{s}{j} \alpha_{j} .
$$

Now (44)-(46) imply (10).

Proof of Theorem 2(a). Assume that $|I|>0$, as otherwise there is nothing to prove.

We shall estimate the probabilities $P\{T=i-l, N=l\}$ for cases $1^{\circ}-4^{\circ}$, listed in the proof of Theorem 1. For this purpose, we make an extensive use of the following well-known relation for the d.f. of the $k$ th smallest order statistic for an i.i.d. sequence $X_{1}, \ldots, X_{n}$ :

$$
F_{X_{(k)}}(x)=\sum_{j=k}^{n}\left(\begin{array}{c}
n \\
j
\end{array}\right)(F(x))^{j}(1-F(x))^{n-j},
$$

where $F$ stands for the marginal d.f. of $X_{1}$.

Suppose that case $1^{\circ}$ is satisfied, i.e. $0<i-l<s-|I|$ and $0<l<|I|$. It follows from (26), (27) and (31) that

$$
\begin{aligned}
& P\{T=i-l, N=l\} \\
\leq & \left(P\left\{q_{(l)} \leq \alpha_{i}\right\}-P\left\{q_{(l+1)} \leq \alpha_{i}\right\}\right)\left(P\left\{r_{(i-l)} \leq \alpha_{i}\right\}-P\left\{r_{(i-l+1)} \leq \alpha_{i}\right\}\right) .
\end{aligned}
$$

By using the fact that the sequence $\left\{q_{n}\right\}$ is i.i.d., as well as (47) and (8), we obtain

$$
\begin{aligned}
& P\left\{q_{(l)} \leq \alpha_{i}\right\}-P\left\{q_{(l+1)} \leq \alpha_{i}\right\} \\
& \quad=\left(\begin{array}{c}
|I| \\
l
\end{array}\right)\left(F_{q}\left(\alpha_{i}\right)\right)^{l}\left(1-F_{q}\left(\alpha_{i}\right)\right)^{|I|-l} \leq\left(\begin{array}{c}
|I| \\
l
\end{array}\right)\left(\alpha_{i}\right)^{l}\left(1-F_{q}\left(\alpha_{i}\right)\right)^{|I|-l} .
\end{aligned}
$$

Similarly, as $\left\{r_{n}\right\}$ is i.i.d., (47) yields

$$
\begin{aligned}
P\left\{r_{(i-l)} \leq \alpha_{i}\right\}-P\left\{r_{(i-l+1)} \leq \alpha_{i}\right\} & \\
& =\left(\begin{array}{c}
s-|I| \\
i-l
\end{array}\right)\left(F_{r}\left(\alpha_{i}\right)\right)^{i-l}\left(1-F_{r}\left(\alpha_{i}\right)\right)^{s-|I|-i+l} .
\end{aligned}
$$


By (48)-(50), we obtain

$$
\begin{aligned}
& P\{T=i-l, N=l\} \\
& \leq\left(\begin{array}{c}
|I| \\
l
\end{array}\right)\left(\alpha_{i}\right)^{l}\left(1-F_{q}\left(\alpha_{i}\right)\right)^{|I|-l}\left(\begin{array}{c}
s-|I| \\
i-l
\end{array}\right)\left(F_{r}\left(\alpha_{i}\right)\right)^{i-l}\left(1-F_{r}\left(\alpha_{i}\right)\right)^{s-|I|-i+l}
\end{aligned}
$$

if $0<i-l<s-|I|$ and $0<l<|I|$.

Now consider case $2^{\circ}$, i.e. $i-l=0$. Then $i=l, 0<i \leq|I|<s$, and (see (36))

$$
P\{T=i-l, N=l\}=P\{T=0, N=i\} \leq P\left\{q_{(i)} \leq \alpha_{i}\right\} P\left\{r_{(1)}>\alpha_{i+1}\right\} .
$$

By (47) and (8), we have

$$
P\left\{q_{(i)} \leq \alpha_{i}\right\} \leq \sum_{j=i}^{|I|}\left(\begin{array}{c}
|I| \\
j
\end{array}\right)\left(\alpha_{i}\right)^{j}\left(1-F_{q}\left(\alpha_{i}\right)\right)^{|I|-j} .
$$

Additionally, as $\left\{r_{n}\right\}$ is i.i.d., we obtain

$$
\begin{aligned}
P\left\{r_{(1)}>\alpha_{i+1}\right\} & =P\left\{r_{1}>\alpha_{i+1}, \ldots, r_{s-|I|}>\alpha_{i+1}\right\} \\
& =\left(P\left\{r_{1}>\alpha_{i+1}\right\}\right)^{s-|I|}=\left(1-F_{r}\left(\alpha_{i+1}\right)\right)^{s-|I|} .
\end{aligned}
$$

Thus, due to (52)-(54),

$$
P\{T=0, N=i\} \leq\left(1-F_{r}\left(\alpha_{i+1}\right)\right)^{s-|I|} \sum_{j=i}^{|I|}\left(\begin{array}{c}
|I| \\
j
\end{array}\right)\left(\alpha_{i}\right)^{j}\left(1-F_{q}\left(\alpha_{i}\right)\right)^{|I|-j} .
$$

Assume now that condition $3^{\circ}$ holds, i.e. $0<i-l<s-|I|$ and $l=|I|$. Then $|I|<i<s, 0<i-|I|<s-|I|$, and (see (38))

$$
\begin{aligned}
P\{T=i-l, N=l\} & =P\{T=i-|I|, N=|I|\} \\
& \leq P\left\{q_{(|I|)} \leq \alpha_{i}\right\}\left(1-P\left\{r_{(i-|I|+1)} \leq \alpha_{i+1}\right\}\right) .
\end{aligned}
$$

Notice that, since $\left\{q_{n}\right\}$ is i.i.d. and (8) holds, we get

$$
P\left\{q_{(|I|)} \leq \alpha_{i}\right\}=P\left\{q_{1} \leq \alpha_{i}, \ldots, q_{|I|} \leq \alpha_{i}\right\}=\left(P\left\{q_{1} \leq \alpha_{i}\right\}\right)^{|I|} \leq\left(\alpha_{i}\right)^{|I|} .
$$

Furthermore, it follows from (47) that

$$
\begin{aligned}
1-P\left\{r_{(i-|I|+1)} \leq\right. & \left.\alpha_{i+1}\right\} \\
& =\sum_{j=0}^{i-|I|}\left(\begin{array}{c}
s-|I| \\
j
\end{array}\right)\left(F_{r}\left(\alpha_{i+1}\right)\right)^{j}\left(1-F_{r}\left(\alpha_{i+1}\right)\right)^{s-|I|-j} .
\end{aligned}
$$

By (56)-(58), we obtain

$$
\begin{aligned}
& P\{T=i-|I|, N=|I|\} \\
& \leq\left(\alpha_{i}\right)^{|I|} \sum_{j=0}^{i-|I|}\left(\begin{array}{c}
s-|I| \\
j
\end{array}\right)\left(F_{r}\left(\alpha_{i+1}\right)\right)^{j}\left(1-F_{r}\left(\alpha_{i+1}\right)\right)^{s-|I|-j} .
\end{aligned}
$$


Finally, suppose that condition $4^{\circ}$ is satisfied, i.e. $i-l=s-|I|$. We have

$$
\begin{aligned}
& \text { (60) } \sum_{i=1}^{\left[\frac{|I|-1}{\gamma}\right] \wedge s} \sum_{\substack{l=[\gamma i]+1 \\
i-l=s-|I|}}^{|I| \wedge i} P\{T=i-l, N=l\} \\
& \leq \sum_{i=(s-|I|)+1}^{s-1} P\{T=s-|I|, N=i-(s-|I|)\}+P\{T=s-|I|, N=|I|\} \\
& \leq \sum_{i=(s-|I|)+1}^{\left[\frac{|I|-1}{\gamma}\right] \wedge(s-1)} P\left\{r_{(s-|I|)} \leq \alpha_{i}\right\}\left(P\left\{q_{(i-(s-|I|))} \leq \alpha_{i}\right\}-P\left\{q_{(i-(s-|I|)+1)} \leq \alpha_{i}\right\}\right) \\
& \quad+P\left\{r_{(s-|I|)} \leq \alpha_{s}\right\} P\left\{q_{(|I|)} \leq \alpha_{s}\right\} .
\end{aligned}
$$

Observe that, due to (47) and (8), we get

$$
\begin{aligned}
& P\left\{q_{(i-(s-|I|))} \leq \alpha_{i}\right\}-P\left\{q_{(i-(s-|I|)+1)} \leq \alpha_{i}\right\} \\
& =\left(\begin{array}{c}
|I| \\
i-s+|I|
\end{array}\right)\left(F_{q}\left(\alpha_{i}\right)\right)^{i-s+|I|}\left(1-F_{q}\left(\alpha_{i}\right)\right)^{s-i} \\
& \leq\left(\begin{array}{c}
|I| \\
i-s+|I|
\end{array}\right)\left(\alpha_{i}\right)^{i-s+|I|}\left(1-F_{q}\left(\alpha_{i}\right)\right)^{s-i} \text {. }
\end{aligned}
$$

Furthermore, we also have

$$
P\left\{r_{(s-|I|)} \leq \alpha_{i}\right\}=\left(F_{r}\left(\alpha_{i}\right)\right)^{s-|I|}, \quad P\left\{q_{(|I|)} \leq \alpha_{s}\right\} \leq\left(\alpha_{s}\right)^{|I|} .
$$

Thus, the relations (60)-(62) yield

$$
\begin{aligned}
& \sum_{i=1}^{\left[\frac{|I|-1}{\gamma}\right] \wedge s} \sum_{l=[\gamma i]+1}^{|I| \wedge i} P\{T=i-l, N=l\} \\
& i-l=s-|I| \\
& {\left[\frac{|I|-1}{\gamma}\right] \wedge(s-1)} \\
& \leq \sum_{i=s-|I|+1}\left(F_{r}\left(\alpha_{i}\right)\right)^{s-|I|}\left(\begin{array}{c}
|I| \\
i-s+|I|
\end{array}\right)\left(\alpha_{i}\right)^{i-s+|I|}\left(1-F_{q}\left(\alpha_{i}\right)\right)^{s-i} \\
& +\left(F_{r}\left(\alpha_{s}\right)\right)^{s-|I|}\left(\alpha_{s}\right)^{|I|} .
\end{aligned}
$$

The relations (25), (51), (55), (59) and (63) imply (11).

Proof of Theorem 2(b). By proceeding analogously to the proof of Theorem 1(b) (see (44)-(46)), we obtain

$$
P\{F D P>\gamma\} \leq \sum_{j=1}^{s} P\left\{q_{(j)} \leq \alpha_{j}\right\} .
$$


It follows from (47) that

$$
P\left\{q_{(i)} \leq \alpha_{i}\right\} \leq \sum_{j=i}^{s}\left(\begin{array}{l}
s \\
j
\end{array}\right)\left(\alpha_{i}\right)^{j}\left(1-F_{q}\left(\alpha_{i}\right)\right)^{s-j} .
$$

The relations (64), (65) imply (12).

Proof of Theorem 3. Obviously, we have

$$
\begin{aligned}
P\{F D P>\gamma\} & =P\{F D P>\gamma, T=0\}+\sum_{t=1}^{s-|I|} P\{F D P>\gamma, T=t\} \\
& =: B_{1}+B_{2} .
\end{aligned}
$$

It is clear that

$$
\begin{aligned}
B_{1} & =P\{1>\gamma, T=0, R>0\}=P\{T=0, R>0\} \\
& =\sum_{j=1}^{s} P\{T=0, R=j\} \\
& =\sum_{j=1}^{|I|} P\{T=0, R=j\}+\sum_{j=|I|+1}^{s} P\{T=0, R=j\} \\
& =\sum_{j=1}^{|I|} P\{T=0, R=j\},
\end{aligned}
$$

where the last equality follows from the fact that

$$
P\{T=0, R=j\}=P\{N=j, R=j\}=0 \quad \text { if } j>|I| .
$$

Notice that, for $j \in\{1, \ldots,|I|\}$,

$$
P\{T=0, R=j\}=P\{N=j, R=j\} \leq P\left\{q_{(j)} \leq \alpha_{j}\right\} .
$$

By Proposition A and assumption (8), we get

$$
P\left\{q_{(j)} \leq \alpha_{j}\right\} \leq \frac{|I|}{j} F_{q}\left(\alpha_{j}\right) \leq \frac{|I|}{j} \alpha_{j} .
$$

Due to (67)-(69), we obtain

$$
B_{1} \leq \sum_{j=1}^{|I|} \frac{|I|}{j} \alpha_{j}
$$

We now estimate the component $B_{2}$ in (66). We have 


$$
\begin{aligned}
B_{2} & =\sum_{t=1}^{s-|I|} P\{F D P>\gamma \mid T=t\} P\{T=t\} \\
& =\sum_{t=1}^{s-|I|} P\left\{\frac{N}{N+T}>\gamma \mid T=t\right\} P\{T=t\} \\
& =\sum_{t=1}^{s-|I|} P\left\{N>t \frac{\gamma}{1-\gamma} \mid T=t\right\} P\{T=t\} \\
& \leq \sum_{t=1}^{s-|I|} P\left\{N>\frac{\gamma}{1-\gamma} \mid T=t\right\} P\{T=t\} .
\end{aligned}
$$

Thus,

$$
B_{2} \leq \sum_{t=1}^{s-|I|} P\{N \geq C(\gamma) \mid T=t\} P\{T=t\} \leq P\{N \geq C(\gamma)\}
$$

where $C(\gamma):=\min ([\gamma /(1-\gamma)]+1,|I|)$. Let $m$ be the smallest index satisfying $p_{(m)}=q_{(C(\gamma))}$. It is easy to check that $C(\gamma) \leq m \leq s-|I|+C(\gamma)$ (see (13) in Lehmann and Romano (2005)). This, (71), Proposition A and assumption (8) yield

$$
\begin{aligned}
B_{2} & \leq P\{N \geq C(\gamma)\} \leq P\left\{q_{(C(\gamma))} \leq \alpha_{m}\right\} \\
& \leq \frac{|I|}{C(\gamma)} F_{q}\left(\alpha_{m}\right) \leq \frac{|I|}{C(\gamma)} \alpha_{m} \leq \frac{|I|}{C(\gamma)} \alpha_{s-|I|+C(\gamma)}
\end{aligned}
$$

The relations (66), (70) and (72) imply (13).

The following two claims are needed for the proofs of our main results.

Proposition A (Proposition 1 in Caraux and Gascuel (1992)). Let $X_{1}, \ldots, X_{n}$ be a set of $n$ identically distributed r.v.'s (with c.d.f. $F$ ) and $F_{X_{(k)}}$ denote the d.f. of the kth smallest order statistic. Then

$$
F_{X_{(k)}}(x) \leq \min \left(\frac{n}{k} F(x), 1\right) .
$$

The next auxiliary result we have used extensively.

Proposition B (Proposition 2 in Caraux and Gascuel (1992)). Let $X_{1}, \ldots, X_{n}$ be a set of $n$ identically distributed r.v.'s (with c.d.f. F) and $F_{X_{(k)}}$ denote the d.f. of the kth smallest order statistic. Then

$$
F_{X_{(k)}}(x) \geq \max \left(1-\frac{n}{n-k+1}(1-F(x)), 0\right) \text {. }
$$

Acknowledgements. We wish to thank an anonymous referee for valuable comments, remarks and suggestions. 


\section{References}

Y. Benjamini and Y. Hochberg (1995), Controlling the false discovery rate: a practical and powerful approach to multiple testing, J. Roy. Statist. Soc. Ser. B 57, 289-300.

G. Caraux and O. Gascuel (1992), Bounds on distribution functions of order statistics for dependent variates, Statist. Probab. Lett. 14, 103-105.

S. Dudoit, J. P. Shaffer and J. C. Boldrick (2003), Multiple hypothesis testing in microarray experiments, Statist. Sci. 18, 71-103.

Y. Hochberg (1988), A sharper Bonferroni procedure for multiple tests of significance, Biometrica 75, 800-802.

S. Holm (1979), A simple sequentially rejective multiple test procedure, Scand. J. Statist. $6,65-70$.

E. L. Lehmann (1957), A theory of some multiple decision problems, Ann. Statist. 28, $1-25$.

E. L. Lehmann and J. P. Romano (2005), Generalizations of the familywise error rate, Ann. Statist. 33, 1138-1154.

K. S. Pollard, M. J. van der Laan, M. D. Birkner and S. Dudoit (2005), Test statistics null distributions in multiple testing: simulation studies and applications to genomics, U. C. Berkeley division of biostatistics working paper series, paper 184 .

J. P. Romano and A. M. Shaikh (2006), On stepdown control of the false discovery proportion, in: Optimality, IMS Lecture Notes Monogr. Ser. 49, Inst. Math. Statist., Beachwood, OH, 33-50.

S. K. Sarkar (1998), Some probability inequalities for ordered $\mathrm{MTP}_{2}$ random variables: a proof of the Simes conjecture, Ann. Statist. 26, 494-504.

J. Storey (2003), The positive false discovery rate: A Bayesian interpretation and the q-value, Ann. Statist. 31, 2013-2035.

Faculty of Applied Informatics and Mathematics

Department of Applied Mathematics

Warsaw University of Life Sciences (SGGW)

Nowoursynowska 159

02-776 Warszawa, Poland

E-mail: marcin_dudzinski@sggw.pl

konrad_furmanczyk@sggw.pl

Received on 12.3.2007;

revised version on 8.10.2009 